\title{
O-6-methylguanine DNA methyltransferase is a favorable biomarker with proliferation suppressive potential in Breast Cancer
}

\author{
Danxia Lin ${ }^{1,2^{*}}$, Yingsheng $\mathrm{Xiao}^{3^{*}}$, Binliang Huang4, Xiao Wu ${ }^{5}$, Chunfa Chen ${ }^{2,6}$, Yuanke Liang7, De Zeng ${ }^{1,2}$ \\ 1. Department of Medical Oncology, Cancer Hospital of Shantou University Medical College, No. 7 Raoping Road, Shantou 515031, PR China. \\ 2. Guangdong Provincial Key Laboratory of Breast Cancer Diagnosis and Treatment, No. 7 Raoping Road, Shantou 515031, PR China. \\ 3. Department of Thyroid Surgery, Shantou Central Hospital, No. 114 Waima Road, Shantou 515031, PR China. \\ 4. Department of Clinical Laboratory Medicine, Cancer Hospital of Shantou University Medical College, Shantou, PR China. \\ 5. Cancer Research Center, Shantou University Medical College, No. 22 Xinlin Road, Shantou 515031, PR China. \\ 6. The Breast Center, Cancer Hospital of Shantou University Medical College, Shantou, PR China. \\ 7. Department of Thyroid and Breast Surgery, the First Affiliated Hospital of Shantou University Medical College, Shantou, Guangdong, PR China. \\ *These authors contributed equally to this work.
}

$\square$ Corresponding author: Dr. De Zeng, Department of Medical Oncology, Cancer Hospital of Shantou University Medical College, 7 Raoping Road, Shantou 515031, PR China; Guangdong Provincial Key Laboratory of Breast Cancer Diagnosis and Treatment, No. 7 Raoping Road, Shantou 515031, PR China. Fax: (+86) 0754-88555844; Tel.: (+86) 0754-88900232; E-mail: dezeng@stu.edu.cn.

() The author(s). This is an open access article distributed under the terms of the Creative Commons Attribution License (https://creativecommons.org/licenses/by/4.0/). See http://ivyspring.com/terms for full terms and conditions.

Received: 2020.03.29; Accepted: 2020.08.15; Published: 2020.09.01

\begin{abstract}

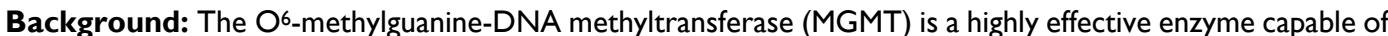
repairing DNA damage to maintain genomic stability. Until recently, reports on the expression and potential role of MGMT in breast cancer remain controversial. This study is intended to elucidate the prognostic significance and potential function of MGMT in breast cancer.

Materials and methods: The immunohistochemistry assay and a series of public databases were utilized to determine the relevance between MGMT expression and clinicopathological characteristics, as well as survival outcomes in patients with breast cancer. The western blotting, QRT-PCR, proliferation, colony formation and transwell assays were used to investigate the potential function of MGMT in breast cancer cells.

Results: The immunohistochemistry analysis and public cancer databases exploration demonstrated that MGMT expression was significantly related to estrogen receptor (ER) positivity in breast cancer. Positive expression of MGMT predicts a longer distant-free survival (DFS) and overall survival (OS) in patients with breast cancer, especially in ER-positive tumor. The mRNA level of MGMT was significantly associated with those of ESR1, GATA3 and FOXA1 in ER-positive breast tumor. Down-regulation of MGMT expression enhanced the proliferative and invasive capacities of breast cancer cells through PTEN/AKT pathway.

Conclusions: MGMT is a favorable biomarker with proliferation suppressive potential in ER-positive breast cancer. Future study on targeted modulation of MGMT in the treatment of breast cancer is warranted.
\end{abstract}

Key words: breast cancer, $\mathrm{O}^{6}$-methylguanine-DNA methyltransferase (MGMT), prognosis, immunohistochemistry (IHC); IRS, immunoreactive score

\section{Introduction}

The $\mathrm{O}^{6}$-methylguanine DNA methyltransferase (MGMT) is a DNA methyltransferase that exerts critical functions in DNA repair [1]. It has been recognized that silencing of MGMT or a decrease in MGMT protein expression could be associated with tumorigenesis [2]. For instance, aberrant promoter methylation resulting in loss of MGMT expression has been demonstrated in approximately $40 \%$ of colorectal cancers and gliomas, as well as in $25 \%$ of lymphomas and non-small cell lung carcinomas [3].

Emerging evidences have demonstrated that breast cancer is virtually a heterogeneous group of diseases with respect to molecular classification and MGMT expression $[4,5]$. However, reports on the 
protein expression profiles and prognostic significance of MGMT in breast cancer remain inconsistent. It has been shown that MGMT expression in breast tumors was up to 4 -fold higher than that in normal counterparts [6, 7]. S. Matsukura and colleagues reported that MGMT deficiency predicted a poor outcome in patients with breast ductal adenocarcinoma [8]. On the contrary, K. Shima and colleagues demonstrated that positive expression of MGMT was correlated to shorter survival in patients with basal-like breast cancer (BLBC) [9].

These conflicting perspectives on the role of MGMT in breast cancer might stem from previous studies that evaluating heterogeneous groups of patients $[10,11]$. Moreover, the majority of existing studies have not presented the pertinence between MGMT expression and overall survival (OS) in patients with breast cancer $[5,8,12,13]$.

In the present study, it was found that MGMT expression was significantly associated with ER positivity in breast cancer. Moreover, MGMT could affect the proliferative and invasive capacities of breast cancer cells through regulating the PTEN/AKT pathway. These results consistently indicated that modulation of MGMT is a potential tactics in the treatment of breast cancer.

\section{Materials and Methods}

\section{Cell lines and cell culture}

Breast cancer cell lines including MDA-MB-231, BT-549, ZR-75-1, T-47D, and MCF-7 were purchased from American Type Culture Collection (ATCC). All the cells were maintained in Dulbecco's Modified Eagle Medium (DMEM) containing 10\% fetal bovine serum (FBS) under the incubation condition of $5 \%$ $\mathrm{CO} 2$ humidity at $37^{\circ} \mathrm{C}$.

\section{Small interfering RNA, Plasmids, and transfection}

The pCMV empty vector and pCMV-MGMT plasmids were designed and provided by Sino Biological (Beijing, China). Small interference RNAs, as shown Table S1, were purchased from GenePharma Company (Suzhou, China). Transfection experiments were performed with addition of Lipofectamine 3000 and P3000 (Life Technology, NY, USA) by following the manufacturer's instruction.

\section{Patient characteristics and tissue samples}

The tissue microarray (TMA) of breast cancer tissues from 275 female patients, none of which had received previous chemotherapy or other anti-tumor treatment before surgery, was collected and provided by Shanghai Outdo Biotech (Shanghai, China). Surgery took place between January 3rd, 2001 and
November 1st, 2008. Patients' clinicopathological features, including gender, age, tumor size, tumor location, clinical stage and follow-up information (ending on July 30th, 2014) were incorporated for the association analysis.

\section{Public cancer databases}

The CCLE database (https://portals. broadinstitute.org/ccle/home) was used to analyze the relative mRNA level of MGMT in a variety of breast cancer cell lines. The Breast Cancer Gene-Expression Miner v4.0 database (http:// bcgenex.centregauducheau.fr/BC-GEM/GEM-requet e.php) was utilized to investigate the association between mRNA levels of MGMT and clinicopathological features of $\mathrm{BC}$, as well as the correlation of MGMT with ESR1, GATA3 and FOXA1.

\section{Western blotting assay}

Cells were suspended in RIPA buffer with protease inhibitors, then the protein extracts were segregated by 10\%SDS-PAGE followed by transferring onto PVDF membrane. Next, the PVDF membrane was incubated with primary antibody overnight (Table S3), and subsequently incubated for 1 hour with appropriate secondary antibodies. Finally, the electrochemiluminescence was used to detect the expression of protein.

\section{qRT-PCR}

TRIzol reagent (Thermo Fisher Scientific, USA) was used to extract the total RNA. The PrimeScript ${ }^{\mathrm{TM}}$ RT Reagent Kit (Takara Bio Inc, Dalian, China) was used for the RNA reverse-transcription into cDNA. Primer sequences for real-time polymerase train reaction (RT-PCR) are listed and shown in Table S2.

\section{Cell viability assay}

Cells were cultured in 96-well plates (1 x 103/well). Then, $10 \mu \mathrm{l}$ of Cell Counting Kit-8 (CCK-8) was added to each well at $0-, 2-, 3-, 4-, 5-, 6-$ and 7-day, with subsequent incubation for 2 hours at $37^{\circ} \mathrm{C}$ in an incubator. The absorbance value was detected at 450 nm.

\section{Transwell assay}

Transfected cells (MCF-7: $4 \times 10^{3}$ cells/well; MDA-MB-231: $2 \times 10^{3}$ cells/well) were placed in the upper chamber in DMEM without serum, and medium containing 10\% serum was added the lower chamber. After 36-hour incubation, the non-migrated cells remain on the upper of each chamber were scraped and removed with a cotton swab, and then, the migrated cells were kept intact and stained with $0.1 \%$ crystal violet. Finally, the migrated cells were counted in every five randomized fields. 


\section{Immunohistochemistry}

After deparaffinage, tissue microarrays were rehydrated in a series of graded ethanol solutions. Then, antigen retrieval was performed in EDTA buffer (1 mmol/l, pH 8.0, Boster Biological Technology Co., Ltd.) for $2.5 \mathrm{~min}$. Next, the tissue microarrays were incubated with a primary antiMGMT monoclonal antibody (Mouse, 1:50 dilution) overnight at $4^{\circ} \mathrm{C}$. The slides were then exposed to $3 \%$ $\mathrm{H} 2 \mathrm{O} 2$ for 10-20 minutes at room temperature to block endogenous peroxidase activity, with subsequent incubation with the secondary antibody for 30 minutes at $37^{\circ} \mathrm{C}$. The DAB (Dako K5007 HRP/DAB+, Rabbit/Mouse) staining time lasted for 10 minutes. Hematoxylin and 1\% hydrochloric acid were used for counterstaining and differentiation. The sections were then washed to blue-black in saturated lithium carbonate and sealed with neutral balsam.

The positive rate of tumor cells was categorized into an array of scores: $0: 0-5 \% ; 1: 6-25 \% ; 2: 26-50 \%$; 3 : $51-75 \%$; and $4: 76-100 \%$. The intensity of immunostaining was defined and scored as: 1 (weak or only cytoplasmic staining); 2 (moderate); and 3 (intense).

The product of the two scores was defined as the immunoreactive score (IRS), which less than 2 were defined as negative (14). The cutoff point for Ki-67 was $14 \%$. Her-2 positive expression was defined as ' +++ ' by IHC assay or ' ++ ' along with gene amplification detected by fluorescent in situ hybridization (FISH) of HER2/CEP17 ratio > 2.0.

\section{Statistical analysis}

The SPSS software (version 22.0) was used for all the statistical analyses in this study. Continuous variables that normally distributed are recorded as the mean \pm standard error $(\bar{X} \pm S)$. The constituent ratio was reported for enumerate data. The median survival time and survival rate were demonstrated for patients' survival outcomes. Chi-square test was used to determine the correlation between MGMT expression and clinicopathological parameters.

Survival was defined as the time from surgery for breast carcinoma until the final follow-up. Breast cancer-related deaths were recorded as events for the purposes of the survival analysis. Patients who were alive at the last follow-up, or who died due to other causes, were censored. Analyses for disease-free survival (DFS) and overall survival (OS) were performed with the Kaplan-Meier method. Univariate analyses of potential prognostic factors were performed with the log-rank test. All tests were two-sided in this study and a $P$ value $<0.05$ was considered to be statistically significant.

\section{Results}

\section{MGMT positive expression was significantly correlated to ER positivity in breast cancer}

We first assessed MGMT expression in tissue microarrays (TMAs) using IHC scored as negative (0), low (1), moderate (2) or high (3) (Fig. 1A). There were $74(26.9 \%)$ cases with MGMT positive expression and 201 cases $(73.1 \%)$ with MGMT negative expression in the 275 breast cancer samples (Fig. 1B). MGMT positivity in the ER-positive group was markedly higher than in the negative group $(p=0.023)$. None of the other clinicopathological parameters analyzed, including age, lymph node metastasis, primary tumor location, tumor grade, tumor size, TNM stage, progesterone receptor expression, Her-2 or Ki-67, were significantly associated with MGMT expression $(p>0.05$, Table 1$)$. The positive rate of MGMT in ER+ breast cancer tissues (32\%) was significantly higher than in ER- counterparts (20\%) (Fig. 1C). Moreover, the positive rate of MGMT was significantly higher detected in Luminal A (33\%) and Luminal B (31\%) subtypes than HER-2 (18\%) and Basal-like (19\%) subtypes (Fig. 1D).

Univariate analysis demonstrated that the 5-year DFS rates were $87.8 \%$ and $75.6 \%(\mathrm{H} R=0.53, p=0.0275)$ in the MGMT-positive and -negative groups, respectively (Fig 1E). The 5-year overall survival (OS) rates were $89 \%$ and $74 \%(\mathrm{HR}=0.54, p=0.033)$ in the MGMT-positive and -negative groups, respectively (Fig. $1 \mathrm{H})$. Subgroup analyses indicated that high mRNA expression of MGMT was significantly correlated to longer survival in ER positive- (DFS: $\mathrm{HR}=0.47, p=0.037$ OS: $\mathrm{H} R=0.39, p=0.0084$ ) (Fig. $1 \mathrm{~F}$ and $1 \mathrm{I})$, but not in ER negative- $\mathrm{BC}$ (DFS: $\mathrm{HR}=1.19, p=0.736$ OS: $H R=0.98, p=0.97$ ) (Fig. $1 \mathrm{G}$ and $1 \mathrm{~J}$ ).

\section{MGMT is particularly high expressed in ER-positive breast tumor and associated with the expression of ESR1, GATA3 and FOXA1}

Through analysis in bc-GenExMiner v4.0 database, we found that the mRNA level of MGMT in ER positive subgroup were significantly higher than ER negative subgroup of breast carcinoma (Fig. 2A). Moreover, the highest MGMT expression was observed in Luminal subtypes of BC (Fig. 2B), all of the group comparisons were shown in Table S4.

Gene correlation targeted analysis revealed that higher mRNA level of MGMT was associated with higher mRNA level of ESR1 ( $r=0.35, p<0.001)$ (Fig 2C), GATA3 $(r=0.37, p<0.001)$ (Fig 2D) and FOXA1 $(r=0.26$, $p<0.001$ ) (Fig. 2E). Correlation maps for all patients among MGMT, ESR1, GATA3 and FOXA1 were showed (Fig. 2F). These results demonstrated the level of MGMT, primarily high expressed in ER-positive 
breast tumor, was positively associated with ESR1, GATA3 and FOXA1.

A
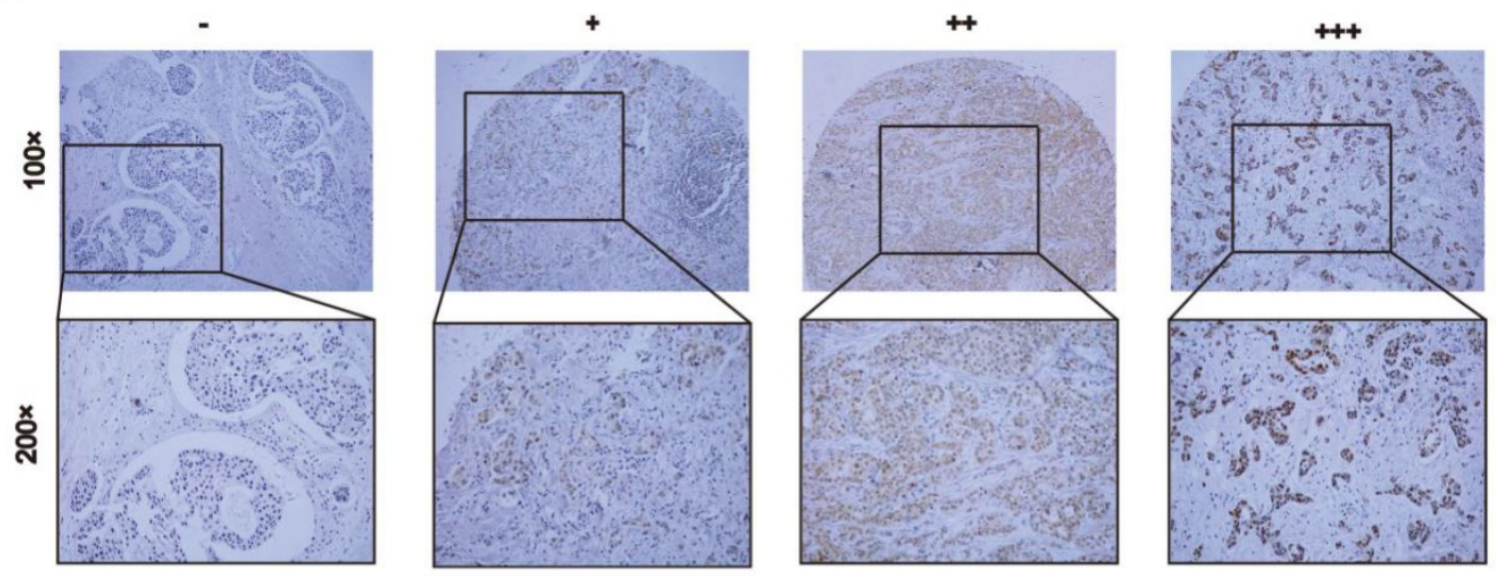

B

MGMT expression

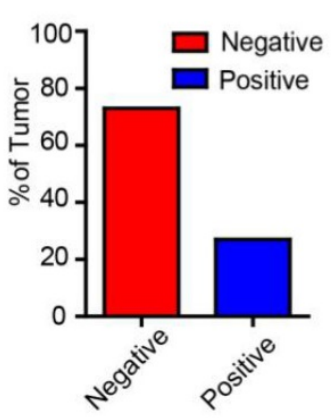

E

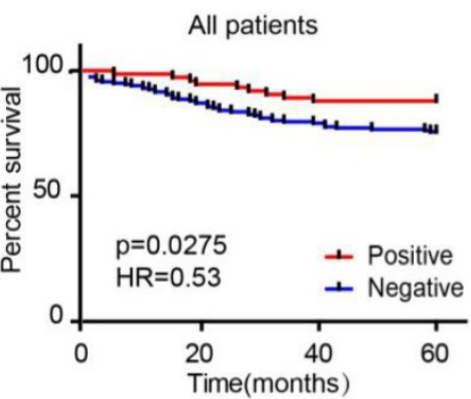

$\mathrm{H}$

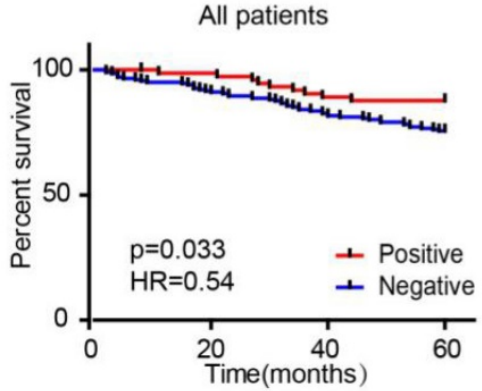

C

MGMT expression/ER status

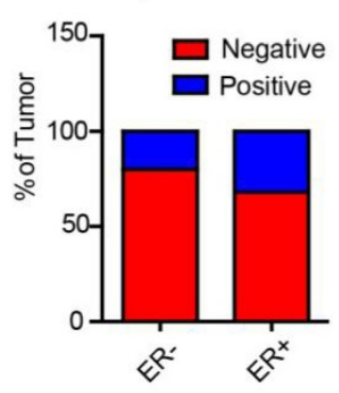

$\mathrm{F}$

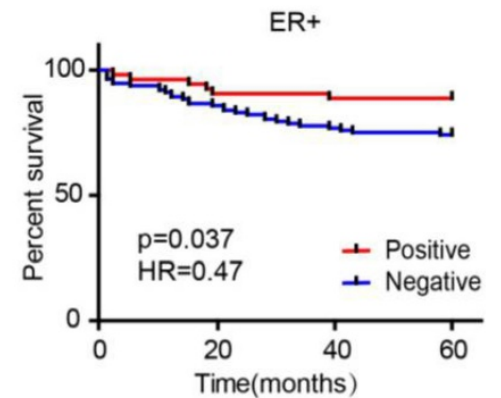

OS

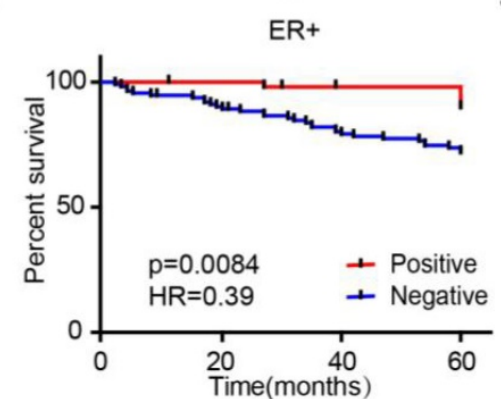

D

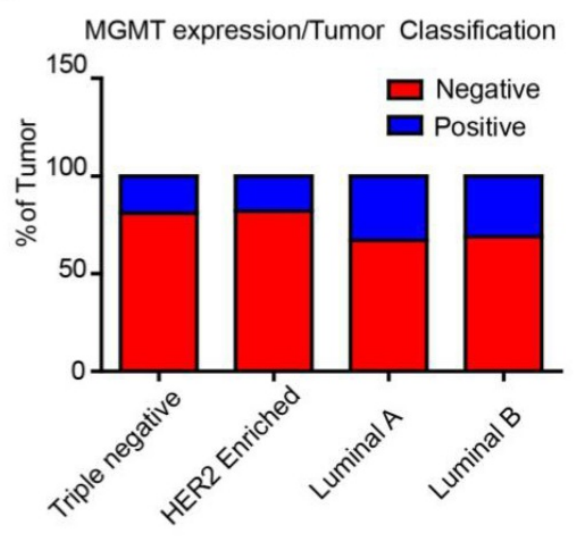

G

ER-

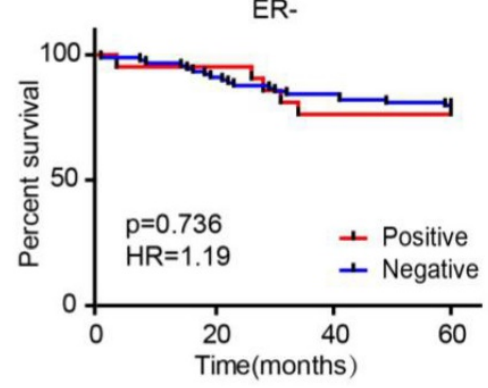

ER-

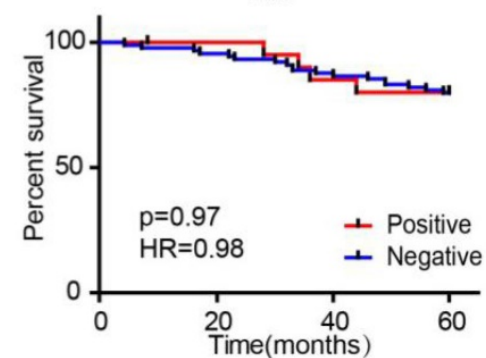

Figure 1. MGMT expression is enriched in ER+ Breast Cancer A. MGMT expression was scored as negative (-), low (+), moderate (++) or high (+++) in breast tumor tissues by using IHC. B. Percentages of total negative or positive samples from the entire cohort are shown. C and D. The TMA samples were classified according to estrogen receptor status or molecular subtype and percentage of MGMT negative versus positive. E-J. High mRNA level of MGMT was associated with longer DFS (E) or OS (H) in all BC patients and in $E R+(F$ and I), but not ER- BC patients ( $G$ and J). 
A

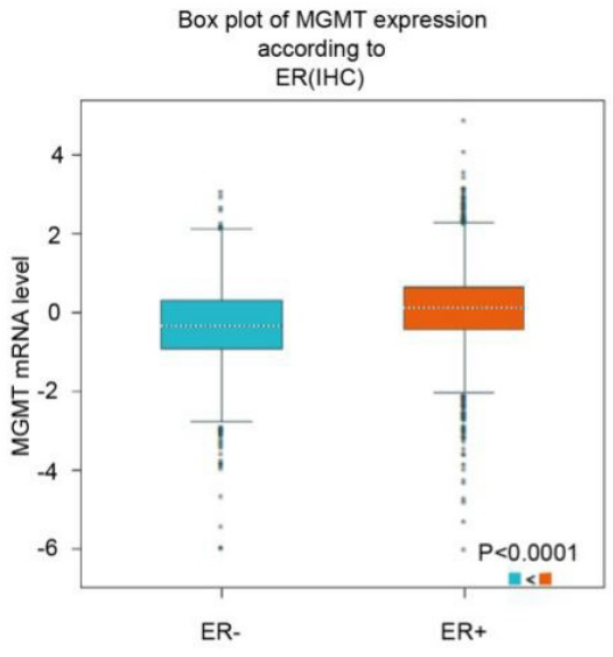

(NO) (1494)

$(3790)$
B

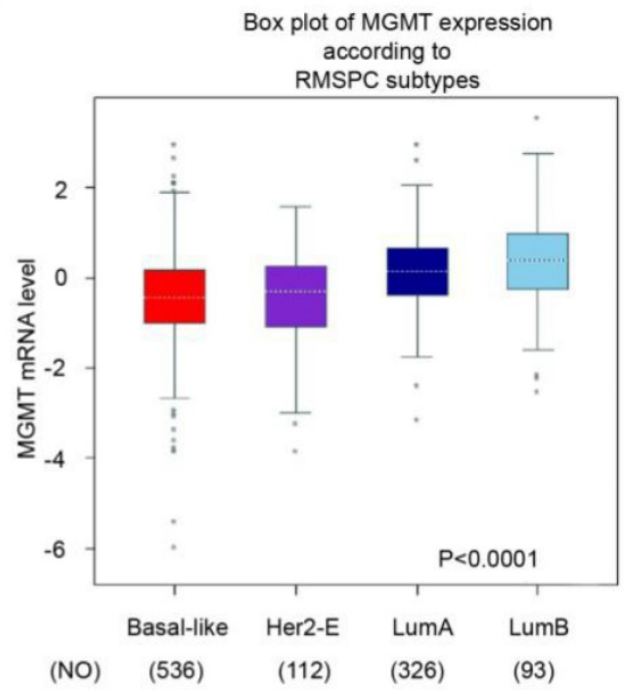

D

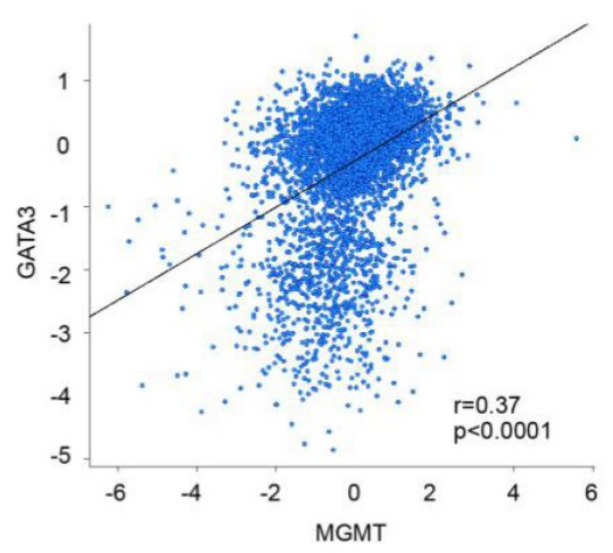

F

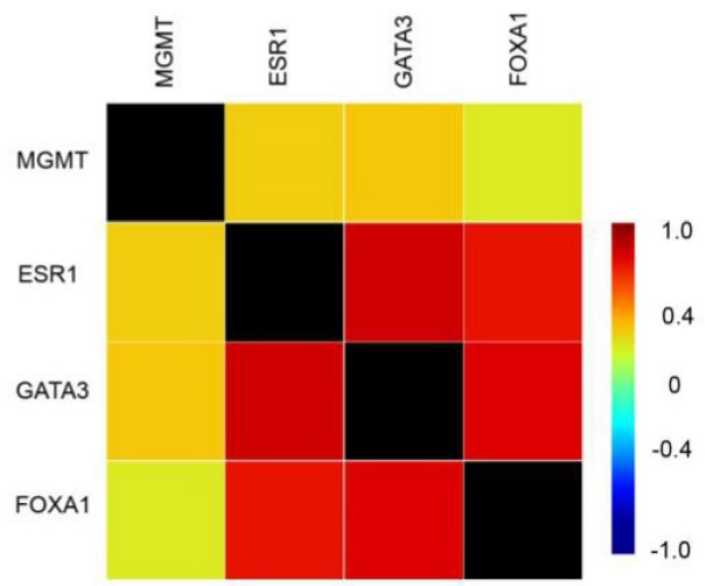

Figure 2. Higher expression of MGMT correlated with high expression of ESR1, GATA3 and FoxA1.A. The mRNA expression level of MGMT in BC patients with ER (-) and ER (+). B. The expression of MGMT in several subtypes of breast cancer patients. C. Gene correlation targeted analysis between MGMT and ESR1. D. Gene correlation targeted analysis between MGMT and GATA3. E. Gene correlation targeted analysis between MGMT and FOXA1. F. Correlation map for all patients among MGMT, ESR1, GATA3 and FOXA1. 
Table 1. Correlation between clinicopathologic parameters and expression of MGMT in breast cancer $(n=275)$

\begin{tabular}{|c|c|c|c|c|}
\hline \multirow[t]{2}{*}{ Clinicopathologic parameters } & \multirow[t]{2}{*}{$\mathrm{n}$} & \multicolumn{2}{|l|}{ MGMT } & \multirow[t]{2}{*}{$P$ value } \\
\hline & & Negative & Positive & \\
\hline Age & & & & 0.398 \\
\hline$<60$ & 171 & 128 & 43 & \\
\hline$\geq 60$ & 104 & 73 & 31 & \\
\hline Lymph node & & & & 0.709 \\
\hline Negative & 124 & 92 & 32 & \\
\hline Positive & 151 & 109 & 42 & \\
\hline Location & & & & 0.056 \\
\hline Left & 120 & 94 & 26 & \\
\hline Right & 155 & 107 & 48 & \\
\hline Grade & & & & 0.959 \\
\hline I-II & 205 & 150 & 55 & \\
\hline III & 70 & 51 & 19 & \\
\hline \multicolumn{5}{|l|}{ Tumor size } \\
\hline T0-T2 & 246 & 178 & 68 & 0.425 \\
\hline T3-T4 & 29 & 23 & 6 & \\
\hline \multicolumn{5}{|l|}{ Staging } \\
\hline I-II & 192 & 143 & 49 & 0.259 \\
\hline III & 83 & 58 & 25 & \\
\hline \multicolumn{5}{|l|}{ ER } \\
\hline Negative & 110 & 88 & 22 & $0.023^{\#}$ \\
\hline Positive & 165 & 113 & 52 & \\
\hline PR & & & & 0.202 \\
\hline Negative & 147 & 111 & 36 & \\
\hline Positive & 128 & 90 & 38 & \\
\hline HER2 & & & & 0.499 \\
\hline Negative & 195 & 143 & 52 & \\
\hline Positive & 80 & 58 & 22 & \\
\hline Ki67 & & & & 0.244 \\
\hline Low & 171 & 122 & 49 & \\
\hline High & 104 & 79 & 25 & \\
\hline
\end{tabular}

\section{MGMT modulates the activity of PTEN/AKT pathway}

In the CCLE public database, we analyzed the expression profile of MGMT in a series of cancer cell lines. It was showed that MGMT expression in ER-positive breast cancer cells was significantly higher than in ER-negative counterparts (Fig. 3A). Next, we performed qRT-PCR assays and found that MGMT was highly expressed in ER-positive breast cancer cell lines, including MCF-7, T-47D and ZR-75-1. In contrast, MGMT expressed a significantly lower level in ER-negative breast cancer cell lines, including MDA-MB-231, BT-549 (Fig. 3B).

Next, we knocked down MGMT via transfection with siMGMT in MCF-7 cells. It was found that the MGMT protein and mRNA expression were successfully down-regulated, as shown in Fig. 3C and 3D. Because siMGMT-1 was more efficiently than siMGMT-2 in knocking-down MGMT, it was used as the main sequence for the following experiments. After transfection with siMGMT in MCF-7 cells, the expression of PTEN decreased, while the expression of pAKT (Ser473), but not total AKT, drastically increased (Fig. 3E). After transfection with pCMV-
MGMT plasmid in MDA-MB-231 cells, the protein level of PTEN increased, while p-AKT (Ser473) expression declined accordingly (Fig. 3F). These results indicated that MGMT was able to regulate the PTEN/AKT pathway.

\section{MGMT suppresses the proliferation and motility of breast cancer cells}

To further investigate the potential function of MGMT in breast cancer, we performed proliferation and colony formation assays in MCF-7 and MDAMB-231 cells. It was found that suppression of MGMT was able to promote cell growth by 1.5-fold and increase the number of colonies by 2 -fold in MCF-7 cells (Fig. 4A and 4B). In contrast, MGMT overexpression could suppress cell proliferation by roughly 2 -fold and decreased the number of colonies by 2 -fold in MDA-MB-231 cells (Fig. 4C and 4D). Next, transwell assays were conducted to evaluate the impact of MGMT on the migratory capacity of breast cancer cells. Suppression of MGMT was able to promote cell migration and invasion in MCF-7 cells (Fig. 4E), while overexpression of MGMT resulted in inhibition of the migration and invasion of MDAMB-231 cells (Fig. 4F). These results revealed that MGMT exerted a pivotal role in regulating the proliferative and migratory abilities of breast cancer cells.

To verify whether the effects of MGMT are dependent on the PI3K/AKT pathway, we performed additional experiments with inhibitor of AKT phosphorylation, As shown in Figure 5, it was found that knocking down MGMT could increase expression of p-AKT, but addition of the AKT phosphorylation inhibitor (LY294002) eliminated this effect (Fig. 5A). What's more, suppression of MGMT was able to promote cell growth and increase the number of colonies in MCF-7 cells. The LY294002 also can weaken the effects of MGMT (Fig. 5B-D).

\section{Discussion}

The $\mathrm{O}^{6}$-methylguanine-DNA methyltransferase (MGMT) is a DNA repair enzyme capable of dissociating alkyl adducts from the $\mathrm{O}^{6}$ position of guanine, thereby providing an essential defense mechanism for the normal cells against malignant transformation [14]. Expression of MGMT protein is discrepant in normal and tumor tissues and has not been well characterized across different tumor types $[15,16]$. A number of studies have demonstrated that MGMT levels were higher in tumors as compared to adjacent normal counterparts [17]. A study of patients with oral squamous cell carcinoma reported by Sawhney and colleagues found a gradual loss of expression of MGMT during the transition from 
hyperplasia to dysplasia, suggesting that a diminution in MGMT expression might be a critical step in oral tumorigenesis [16].

Promoter methylation of MGMT is a critical mechanism of MGMT silencing, resulting in the loss of MGMT expression or MGMT negativity [18]. A meta-analysis by Nairui and colleagues suggested that MGMT promoter methylation was significantly associated with decreased expression of MGMT

A

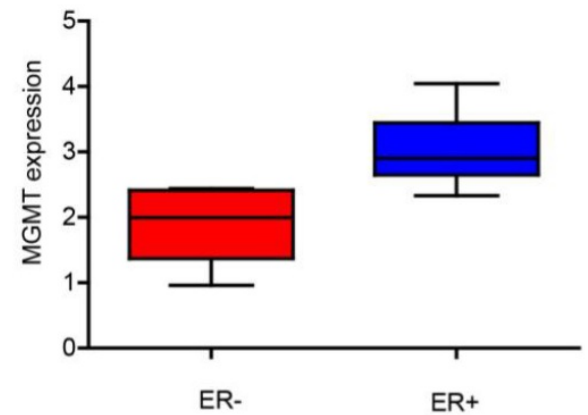

C

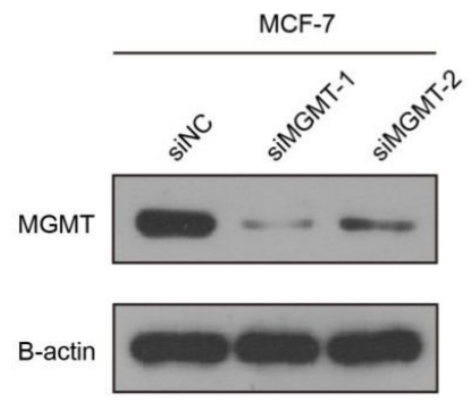

E

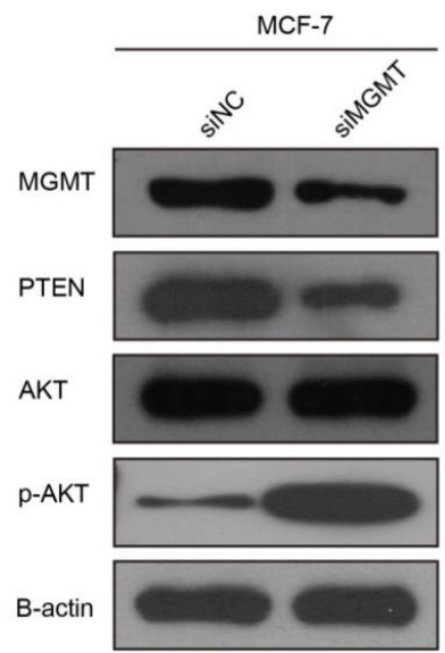

protein and a lack of estrogen receptor (ER) expression [15]. In the present study, MGMT expression was also found to be significantly associated with ER positivity in breast cancer. However, the expression of MGMT was not associated other clinicopathological parameters, including age, lymph node status, tumor location, tumor size, PR status, Ki-67 index and Her-2 status.

B

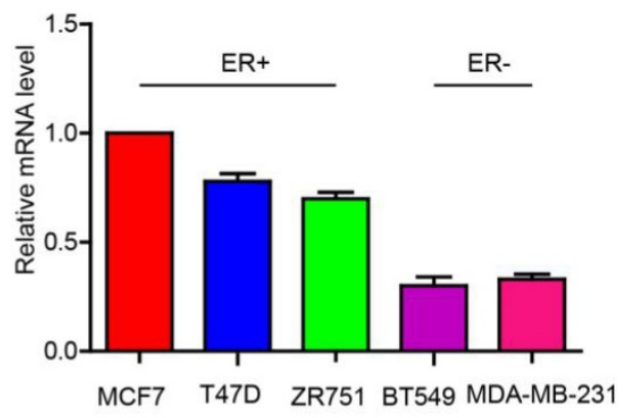

D

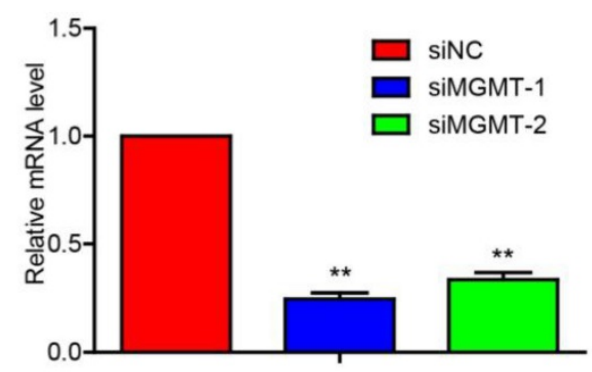

$\mathrm{F}$

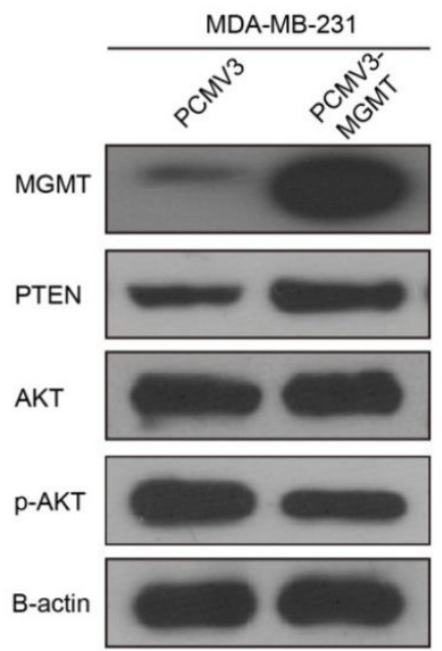

Figure 3. MGMT can modulate the activity of PTEN/AKT pathwayA. MGMT expression in ER+ or ER- status of breast cancer cells using CCLE analysis. B. MGMT expression levels in various human breast cancer cell lines were tested via QRT-PCR. C and D. MCF-7 cells were transfected with two different sequences of MGMT siRNAs. E. Protein levels of MGMT, PTEN, AKT and p-AKT were detected by Western-blot analysis in siMGMT transfected MCF-7 cells, PCMV-MGMT transfected MDA-MB-231 and their control cells. 
A

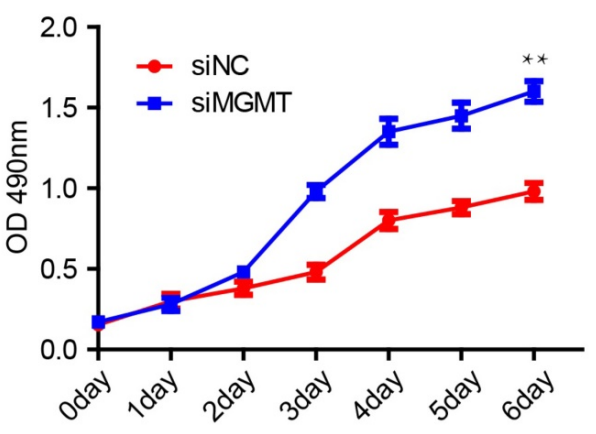

C

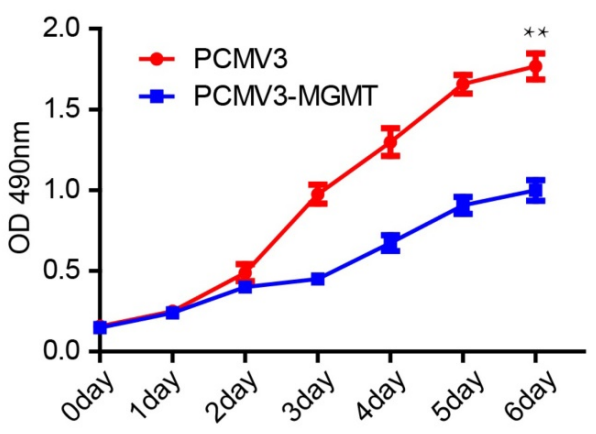

E
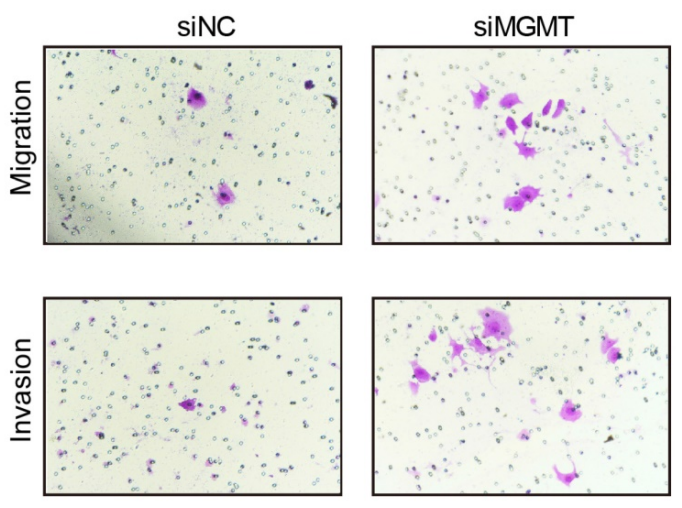

F
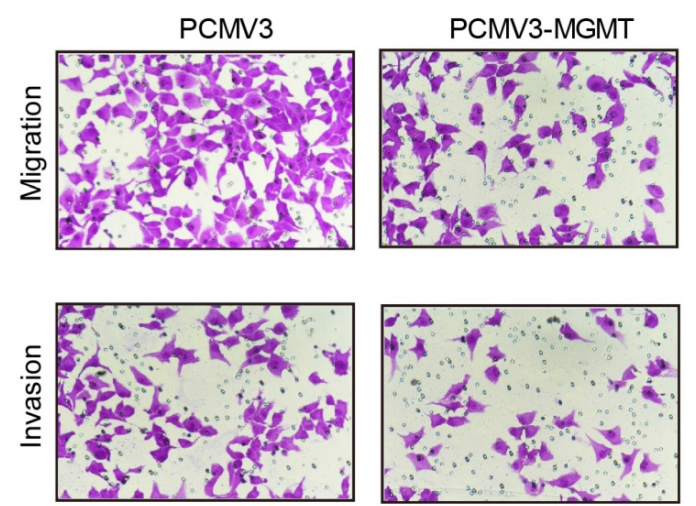

B

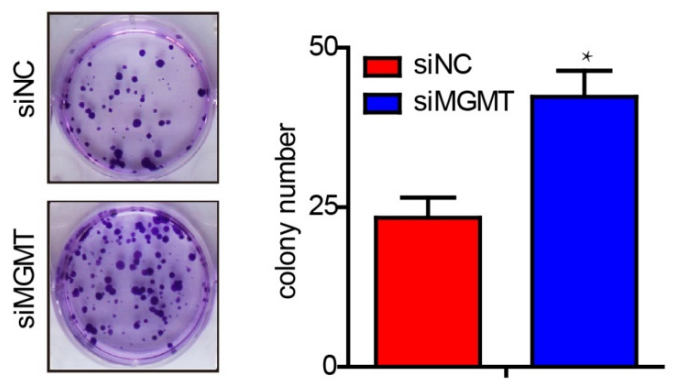

D
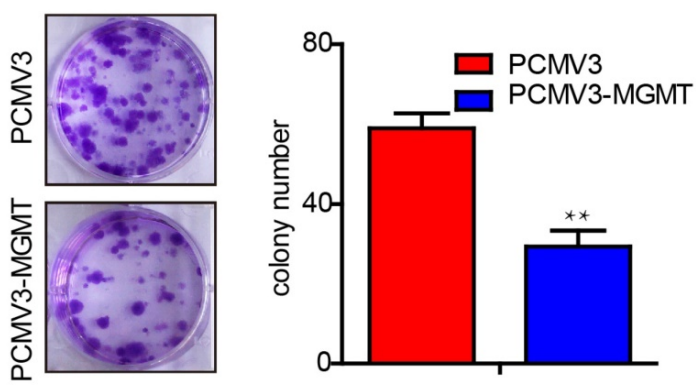
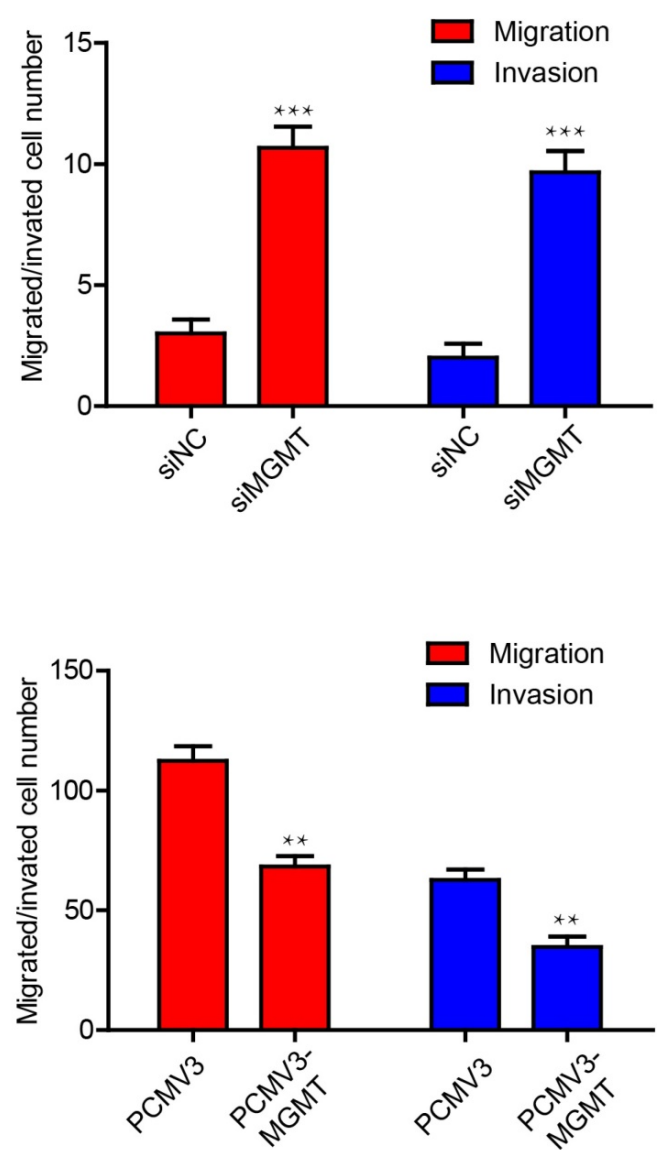

Figure 4. MGMT expression could suppress the cell proliferation and migration in breast cancer cells. A and B. Inhibition of MGMT could enhance cell growth and colony formation in MCF-7 cells. C and D. Overexpression of MGMT can suppress cell growth and colony formation in MDA-MB-231 cells. E. The cell migration and invasion ability was evaluated using a transwell assay in MCF-7 cells transferred with siMGMT and its control. F. The cell migration and invasion ability was evaluated using a transwell assay in MDA-MB-231 cells transferred with PCMV-MGMT and its control. 
A

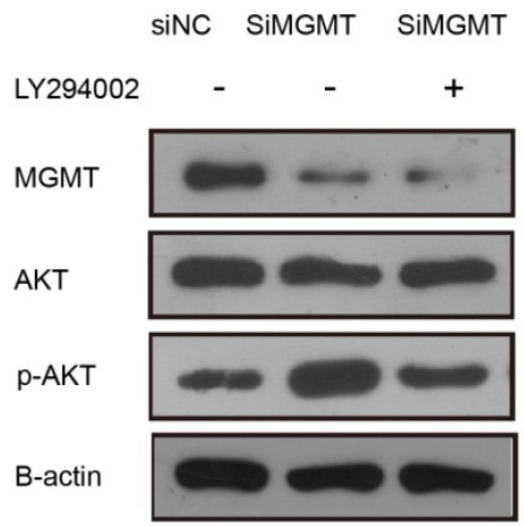

C

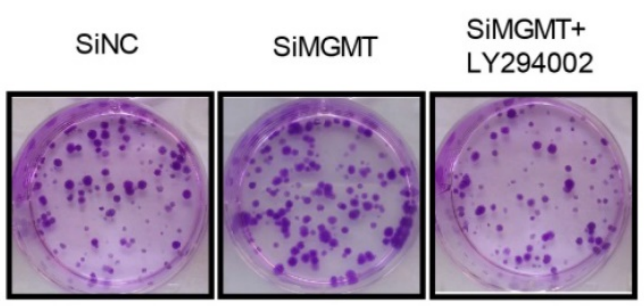

B

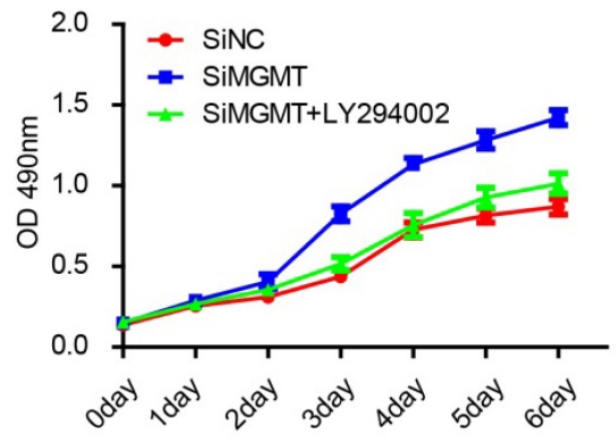

D

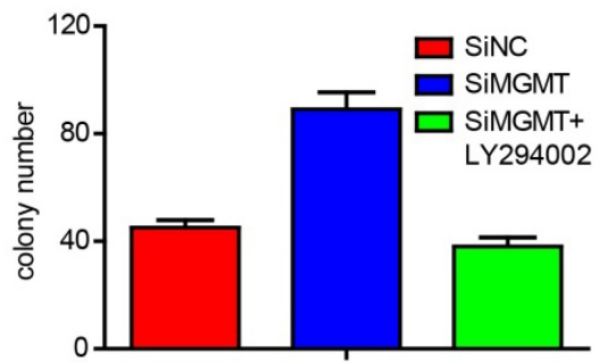

Figure 5. The inhibitor of the AKT phosphorylation (LY294002) can weaken the effects of MGMT on the PI3K/AKT pathway. A. The protein levels of MGMT, AKT and p-AKT were detected by Western-blot analysis in siMGMT transfected MCF-7 cells, with addition of LY294002 and their control cells. B-D. The cell growth curves and colony formation of MCF-7 cells transfected with siMGMT or siMGMT with LY294002.

Survival analysis demonstrated that MGMT positive expression was associated with a better DFS and OS in patients with breast cancer. This result was consistent with the study by Cayre and colleagues, indicating that low MGMT expression was significantly related to poor survival [5]. MGMT negativity resulting from methylation of MGMT may contribute to alkylating agent sensitivity in breast cancer, thereby leading to a longer DFS for patients receiving postoperative adjuvant chemotherapy [19]. Moreover, we found that MGMT gene was positively associated with the classical luminal epithelial regulation genes, including ESR1, GATA3 and FoxA1. It is, therefore, postulated that MGMT might act as a potential modulator of breast epithelial phenotype and a pivotal prognostic biomarker for patients with BC.

In addition, we found that silencing of MGMT was able to inhibit the PTEN expression and increase the p-AKT level, while overexpression of MGMT could increase the PTEN expression and decrease the p-AKT level. These findings confirm the perspective of Neto's study, which suggested that there was a positive correlation between the expressions of
MGMT and PTEN [13]. Moreover, Zhang, L. H. and colleagues have reported that TRIM24 can regulate MGMT expression through PI3K/Akt/NF-kB signaling transduction [20]. We hypothesized that MGMT can negatively regulate Akt via up-regulation of PTEN in breast cancer.

MGMT has been reported to be associated with tumor growth and metastasis in patients with glioblastoma and cholangiocarcinoma. Chen, J. and colleagues have found that knockdown of MGMT induced cell cycle entry by down-regulating p21, p27, and Cyclin E expression, thus promoting ICC proliferation [21, 22]. A study from Li, C. et al. showed that silencing of MGMT increased the invasive and metastatic potentials of glioma cells via up-regulating MMP2 [23]. However, the role of MGMT in breast cancer was poorly elucidated. Our study demonstrated that silencing of MGMT could promote the proliferative and invasive capacities of MCF-7 cells. MGMT overexpression was able to suppress the proliferative and invasive capacities of MDA-MB-231 cells.

In summary, MGMT expression was significantly associated with ER positivity in breast 
cancer. Positive expression of MGMT predicted a better DFS and OS in patients with breast cancer, especially in ER-positive breast cancer. Moreover, MGMT expression could affect the proliferative and invasive abilities of breast cancer cells through the PTEN/AKT pathways (Fig. 6). Future studies on the specific role of MGMT in the pathogenesis and development of breast cancer, particularly in ERpositive subtype, are warranted.

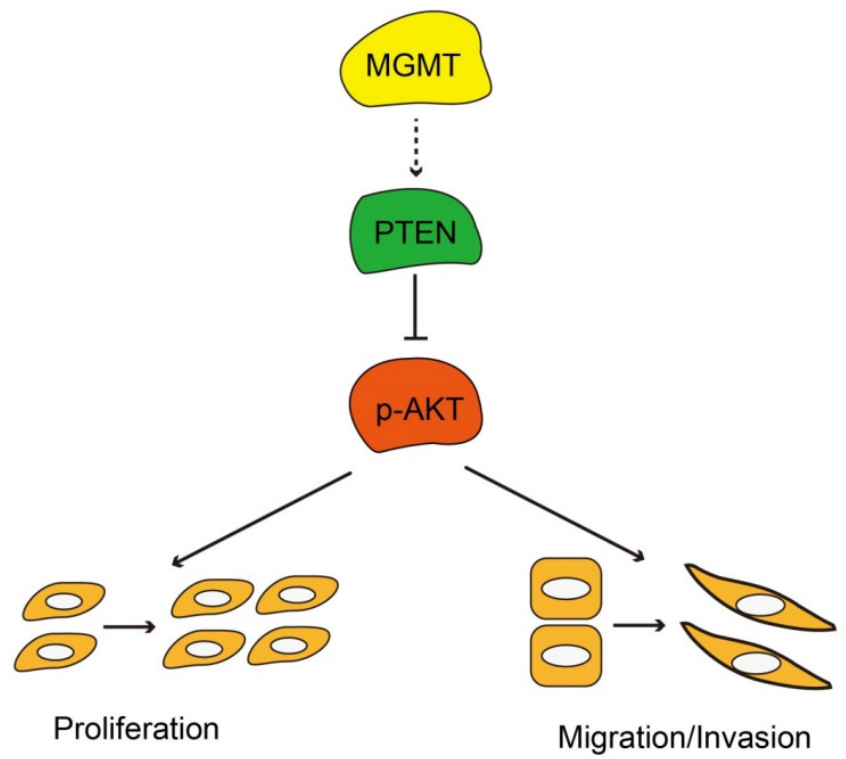

Figure 6. Schematic diagram showing MGMT suppressed the activity of AKT pathway via up-regulation of PTEN, thus suppressing proliferation and migration / invasion.

\section{Abbreviations}

IHC, immunohistochemistry; MGMT, $\mathrm{O}^{6}-$ methylguanine-DNA methyltransferase; TMA, tissue microarray; FISH, fluorescent in situ hybridization; Her-2, human epidermal growth factor receptor-2; ER, estrogen receptor; $\mathrm{PR}$, progesterone receptor; $\mathrm{BLBC}$, basal-like breast cancer; DFS, disease-free survival; OS, survival overall.

\section{Supplementary Material}

Supplementary tables.

http://www.jcancer.org/v11p6326s1.pdf

\section{Acknowledgments}

This work was partly supported by grants from the Guangdong Basic and Applied Basic Research Foundation, China (no. 2018A030313562), Shantou Municipal health science and technology project, Guangdong, China (no. 190819145262877 \& No. 190822165263884), 2019 Special funds for science and technology of Guangdong province, China. "Dengfeng Project" for the construction of high-level hospitals in Guangdong Province-the First Affiliated Hospital of Shantou University Medical College Supporting Funding, Department of Education of Guangdong Province (2019KQNCX035), Guangdong basic and applied basic research foundation (2019A1515110953), Youth Scientific Research Fund of Cancer Hospital affiliated to Shantou University Medical College (no. 2020A003 \& no. 2020A007), 2018 Special Fund for Science and Technology Innovation Strategy of Guangdong Province (no. Shantou Government 2018-49), 2019 Science and Technology Special Fund of Guangdong Province (no. Shantou Government 2019-132), Interdisciplinary project of Li-Ka-Shing Foundation, (no. 2020LKSFG05C), and National Natural Science Foundation of China (no. 81602345).

\section{Authors' contributions}

DXL and DZ conceived and designed the project. DXL, YSX and BLH analyzed the data and prepared the figures and tables. XW performed the immunohistochemistry assay. DXL, YSX, YKL and DZ wrote the manuscript. DZ approved the final version to be submitted.

\section{Ethics approval and consent to publish}

All of the protocols using human specimens were approved by the Shanghai Jiao Tong University, and informed consent was obtained from all of the subjects.

\section{Availability of data and materials}

The datasets used and/or analyzed during the current study are available from the corresponding author on reasonable request.

\section{Ethical approval}

All patients involved in this study have written informed consent.

\section{Competing Interests}

The authors have declared that no competing interest exists.

\section{References}

1. Asiaf A, Ahmad ST, Malik AA, Aziz SA, Rasool Z, Masood A et al. Protein expression and methylation of MGMT, a DNA repair gene and their correlation with clinicopathological parameters in invasive ductal carcinoma of the breast. Tumour Biol 2015; 36: 6485-6496.

2. Sharma S, Salehi F, Scheithauer BW, Rotondo F, Syro LV, Kovacs K. Role of MGMT in tumor development, progression, diagnosis, treatment and prognosis. Anticancer Res 2009; 29: 3759-3768.

3. Esteller M, Hamilton SR, Burger PC, Baylin SB, Herman JG. Inactivation of the DNA repair gene O6-methylguanine-DNA methyltransferase by promoter hypermethylation is a common event in primary human neoplasia. Cancer Res 1999; 59: 793-797.

4. Taherian-Fard A, Srihari S, Ragan MA. Breast cancer classification: linking molecular mechanisms to disease prognosis. Brief Bioinform 2015; 16: 461-474.

5. Cayre A, Penault-Llorca F, De Latour M, Rolhion C, Feillel V, Ferriere JP et al. $\mathrm{O}(6)$-methylguanine-DNA methyl transferase gene expression and prognosis in breast carcinoma. Int J Oncol 2002; 21: 1125-1131. 
6. Wani G, D'Ambrosio SM. Expression of the O6-alkylguanine-DNA alkyltransferase gene is elevated in human breast tumor cells. Anticancer Res 1997; 17: 4311-4315.

7. Citron M, Schoenhaus M, Rothenberg H, Kostroff K, Wasserman P, Kahn L et al. O6-methylguanine-DNA methyltransferase in normal and malignant tissue of the breast. Cancer Invest 1994; 12: 605-610.

8. Matsukura S, Miyazaki K, Yakushiji H, Ogawa A, Harimaya K, Nakabeppu Y et al. Expression and prognostic significance of O6-methylguanine-DNA methyltransferase in hepatocellular, gastric, and breast cancers. Ann Surg Oncol 2001; 8: 807-816.

9. Shima K, Morikawa T, Baba Y, Nosho K, Suzuki M, Yamauchi M et al. MGMT promoter methylation, loss of expression and prognosis in 855 colorectal cancers. Cancer Causes Control 2011; 22: 301-309.

10. Clemons MJ, Bibby MC, El Teraifi H, Forster G, Kelly J, Banerjee S et al. Heterogeneity of O6-alkylguanine DNA-alkyltransferase expression in human breast tumours. Br J Cancer 2002; 86: 1797-1802.

11. Isono S, Fujishima M, Azumi T, Hashimoto Y, Komoike Y, Yukawa M et al. O6-methylguanine-DNA methyltransferase as a prognostic and predictive marker for basal-like breast cancer treated with cyclophosphamide-based chemotherapy. Oncol Lett 2014; 7: 1778-1784

12. Osanai $T$, Takagi $Y$, Toriya $Y$, Nakagawa $T$, Aruga $T$, Iida $S$ et al. Inverse correlation between the expression of O6-methylguanine-DNA methyl transferase (MGMT) and p53 in breast cancer. Jpn J Clin Oncol 2005; 35: $121-125$

13. Neto JC, Ikoma MM, Carvalho KC, Vassallo J, De Brot M, Gobbi H et al. MGMT and PTEN as potential prognostic markers in breast cancer. Exp Mol Pathol 2012; 92: 20-26.

14. Pegg AE. Repair of O(6)-alkylguanine by alkyltransferases. Mutat Res 2000; 462: 83-100.

15. An N, Shi Y, Ye P, Pan Z, Long X. Association Between MGMT Promoter Methylation and Breast Cancer: a Meta-Analysis. Cell Physiol Biochem 2017; 42: 2430-2440.

16. Sawhney M, Rohatgi N, Kaur J, Gupta SD, Deo SV, Shukla NK et al. MGMT expression in oral precancerous and cancerous lesions: correlation with progression, nodal metastasis and poor prognosis. Oral Oncol 2007; 43: 515-522.

17. Bobola MS, Alnoor M, Chen JY, Kolstoe DD, Silbergeld DL, Rostomily RC et al. O(6)-methylguanine-DNA methyltransferase activity is associated with response to alkylating agent therapy and with MGMT promoter methylation in glioblastoma and anaplastic glioma. BBA Clin 2015; 3: 1-10.

18. Tserga A, Michalopoulos NV, Levidou G, Korkolopoulou P, Zografos G, Patsouris E et al. Association of aberrant DNA methylation with clinicopathological features in breast cancer. Oncol Rep 2012; 27: 1630-1638.

19. Gerson SL. MGMT: its role in cancer aetiology and cancer therapeutics. Nat Rev Cancer 2004; 4: 296-307.

20. Zhang LH, Yin AA, Cheng JX, Huang HY, Li XM, Zhang YQ et al. TRIM24 promotes glioma progression and enhances chemoresistance through activation of the PI3K/Akt signaling pathway. Oncogene 2015; 34: 600-610.

21. Chen J, Li Z, Chen J, Du Y, Song W, Xuan Z et al. Downregulation of MGMT promotes proliferation of intrahepatic cholangiocarcinoma by regulating p21. Clin Transl Oncol 2020; 22: 392-400.

22. Li C, Deng L, Shen H, Meng O, Oian A, Sang H et al. O-6-methylguanine-DNA Methyltransferase Inhibits Gastric Carcinoma Cell Migration and Invasion by Downregulation of Matrix Metalloproteinase 2. Anticancer Agents Med Chem 2016; 16: 1125-1132.

23. Yi GZ, Liu YW, Xiang W, Wang H, Chen ZY, Xie SD et al. Akt and beta-catenin contribute to TMZ resistance and EMT of MGMT negative malignant glioma cell line. J Neurol Sci 2016; 367: 101-106. 